\title{
Relative Quantitative Comparison between Lipotoxicity and Glucotoxicity Affecting the PARP-NAD-SIRT1 Pathway in Hepatocytes
}

\author{
Jing Panga,c Chao Xib,c Junhua Jin ${ }^{\mathrm{a}}$ Yiwen Han ${ }^{\mathrm{a}}$ Tie-mei Zhang ${ }^{\mathrm{a}}$
}

aThe Key Laboratory of Geriatrics, Beijing Hospital \& Beijing Institute of Geriatrics, Ministry of Health, ${ }^{b}$ College of Life Sciences, Beijing Normal University, These authors contributed equally to this work

\section{Key Words}

Cytotoxicity • Free fatty acids • Hyperglycemia • Hepatocytes $•$ Poly (ADP)-ribose polymerase

\begin{abstract}
Background/Aims: Insulin resistance in type 2 diabetes results from a combination of hyperglycemia and elevated free fatty acid (FFA) concentrations. However, the individual effects of glucotoxicity and lipotoxicity on cell function have not been determined. Methods: To compare the effects of increased FFAs and glucose levels on the PARP-NAD-SIRT1 pathway, which modulates insulin sensitivity, we cultured HepG2 hepatocytes with 300 or $500 \mu \mathrm{M}$ oleic acid (OA) or $30 \mathrm{mM}$ glucose for 1-4 days. PARP activity, NAD level, SIRT1 expression and insulin receptor phosphorylation were determined. Results: PARP activity was higher while NAD level and SIRT1 expression were lower in OA-treated cells than in control cells. Insulin receptor phosphorylation in response to insulin stimulation was attenuated under OA stimulation. Compared to glucose, OA produced a more rapid effect on the PARP-NAD-SIRT1 pathway in HepG2 cells. The reduction in SIRT1 expression and insulin receptor phosphorylation was similar in cells treated with $500 \mu \mathrm{M}$ OA for 1 day and those treated with $30 \mathrm{mM}$ glucose for 4 days. In addition to PARP activation, the LXR $\alpha$ activator T0901317 also affected SIRT1 expression. Conclusion: FFAs modulated cellular function through multiple ways, and induced more rapid and more potent cytotoxicity than glucose.
\end{abstract}

Copyright (C) 2013 S. Karger AG, Basel 


\section{Introduction}

Excess caloric intake and nutrient availability lead to alterations in glucose and lipid metabolism and have been linked to the pathophysiology of type 2 diabetes [1]. Glucose and lipid metabolism are closely interrelated, and it is difficult to distinguish between the effects of glucotoxicity and lipotoxicity in vivo. Changes in the concentration of either glucose or lipid can induce different cellular reactions contributing to tissue dysfunction. In vitro cellular toxicity has been observed in the presence of glucose accumulation as well as fatty acid accumulation [2,3], but there has been no comparison between glucotoxicity and lipotoxicity. Such a comparison could improve our understanding of the extent of cellular damage under different metabolic conditions in vivo.

Our previous study elucidated a novel pathway involved in glucotoxicity: excessive glucose concentrations activated poly (ADP)-ribose polymerase (PARP), leading to the depletion of nicotinamide adenine dinucleotide (NAD) and inhibition of SIRT1 expression (the PARP-NAD-SIRT1 pathway) in the liver [4]. PARP, a DNA repair enzyme, is activated in response to localized DNA strand breaks and utilizes NAD to catalyze the poly(ADP-ribosyl) ation of acceptor proteins [5]. In diabetes, there are abnormalities of lipid metabolism in addition to an increase in free fatty acid (FFA) concentrations [6]. While it is clear that FFAs can also induce DNA damage [7], the effect of FFAs on PARP activation has not been investigated.

SIRT1, an $\mathrm{NAD}^{+}$-dependent deacetylase, regulates many cellular pathways involved in glucose and lipid homeostasis, and has been suggested to modulate hepatic glucose metabolism by interacting with PGC1a [8]. In white adipocytes, SIRT1 promotes fat mobilization by repressing peroxisome proliferator-activated receptor gamma [9]. Recently, it was reported that SIRT1 is also involved in insulin pathway modulation by increasing insulin receptor phosphorylation [10]. Thus, SIRT1, as a common regulatory element in both lipid and glucose metabolic pathways, provides us with a target to compare the extent of cellular damage caused by glucose and lipids.

In this study, we compared the toxic effects of FFAs and glucose on the PARP-NADSIRT1 pathway in hepatocytes. In addition, we discuss the possible mechanisms underlying the FFA-mediated modulation of SIRT1 expression by comparing FFA toxicity with glucose toxicity. Our findings extend our understanding of the specific roles of glucotoxicity and lipotoxicity in the pathophysiology of diabetes.

\section{Materials and Methods}

\section{Cell culture}

Human hepatoma HepG2 cells were obtained from the Concord Cell Center (Peking Union Medical College, Beijing, China). The cells were cultured in Eagle minimum essential medium (MEM; Invitrogen, Carlsbad, CA) supplemented with 10\% fetal bovine serum (PAA Laboratories, Pasching, Austria) and 5.5 $\mathrm{mM}$ glucose (control) or $30 \mathrm{mM}$ glucose (high glucose concentration). The cell culture conditions were a humidified atmosphere with $5 \% \mathrm{CO}_{2}$ and a temperature of $37^{\circ} \mathrm{C}$. For FFA treatment, cells were cultured in MEM containing $5.5 \mathrm{mM}$ glucose and stimulated with $300 \mu \mathrm{M}$ or $500 \mu \mathrm{M}$ oleic acid (OA; Sigma-Aldrich, St. Louis, MO), which was dissolved in $11 \%$ bovine serum albumin (BSA; stock solution $10 \mathrm{mM}$ ). Control cells were stimulated with BSA alone. T0901317 (Sigma-Aldrich) was dissolved with dimethyl sulfoxide (DMSO; stock solution $10 \mathrm{mM}$ ) and used to determine SIRT1 expression. HepG2 cells were treated with 5 $\mu \mathrm{M}$ T0901317 for 1 day; control cells were treated with DMSO alone.

\section{Western blot}

Samples were separated by $10 \%$ sodium dodecyl sulfate polyacrylamide gel electrophoresis, transferred to a polyvinylidene fluoride membrane and blocked with 5\% milk in Tris-buffered saline with Tween. The samples were then incubated overnight at $4^{\circ} \mathrm{C}$ with the following antibodies: anti- 
SIRT1 (Upstate, Temecula, CA), anti-LXR $\alpha$ (Abcam, Hong Kong), anti-poly(ADP-ribose) (PAR; Trevigen, Gaithersburg, MD), anti-PARP1, anti-phospho-AMPK $\alpha$ (Thr172), anti-AMPK $\alpha$, anti-insulin receptor $\beta$ (InsR $\beta$ ) and anti-phospho-InsR $\beta$ (p-Tyr1150/1151-InsR) (Cell Signaling Technology, Danvers, MA). Bound antibodies were detected with horseradish peroxidase (HRP)-linked secondary antibodies (Jackson ImmunoResearch Laboratories, West Grove, PA) and visualized using enhanced chemiluminescence. To determine if insulin receptors were phosphorylated, we stimulated cells with $100 \mathrm{nM}$ insulin for 20 min before lysis.

\section{Quantitative real-time polymerase chain reaction}

Total RNA was extracted from HepG2 cells using Trizol (Invitrogen), and cDNA was synthesized from $2 \mu \mathrm{g}$ total RNA using M-MuLV reverse transcriptase (New England Biolabs, Beverly, MA). Real-time polymerase chain reaction (PCR) was performed in a Lightcycler (Bio-Rad, Hercules, CA) using the SYBR Green Master Mix Kit. PCR primers were constructed for SIRT1 and LXR $\alpha$ based on published nucleotide sequences of human gene regions. The following primers were used: human $L X R \alpha$, forward 5'-GCC GAG TTT GCC TTG CTC A-3' and reverse 5'-TCC GGA GGC TCA CCA GTT TC-3'; human SIRT1, forward 5'- GCG ATT GGG TAC CGA GAT AAC-3' and reverse 5'-GGC CTT GGA GTC CAG TCA CTA-3'. The reaction specificity was checked by analysis of PCR products in an agarose gel and by analysis of dissociation curves. To assess the amplification efficiency of different pairs of primers, a two-fold serial dilutions of control cDNA were amplified by real-time PCR using primers for $L X R \alpha$, SIRT1 and HPRT. The $2^{-\Delta \Delta C}{ }_{T}$ method was used to analyze the relative changes in gene expression. HPRT served as an internal control, and the expression of the transcripts was quantified as a ratio of HPRT expression.

\section{NAD examination}

Intracellular NAD content was measured using the $\mathrm{NAD}^{+} / \mathrm{NADH}$ quantification kit (Bioassay systems, Hayward, CA), according to the manufacturer's instructions.

\section{Cell viability}

Water-soluble tetrazolium salt-1 (Beyotime), which is an alternative to MTT, was added to 96-well plate; subsequently, the plate was incubated for $2 \mathrm{~h}$ at $37^{\circ} \mathrm{C}$. Cell viability was measured at $450 \mathrm{~nm}$, using a microplate reader (Spectramax 190, Molecular Devices).

\section{Statistical analysis}

Data were expressed as mean and standard deviation. The distribution of variables was analyzed with the data histograms, and a normal distribution was tested using the Kolmogorov-Smirnov test. Comparisons between two mean values were made using the independent-samples $t$-test. For multiple comparisons among different groups of data, significant differences were determined using one-way analysis of variance and the SPSS software for Windows 11.0. Differences between values were considered significant at $\mathrm{P}<0.05$.

\section{Results}

Effects of $O A$ and glucose on PARP activity and intracellular NAD content

We determined the total poly(ADP-ribosyl)ated protein content by western blotting and presented the results as PARP activity. The PARP activity in cells incubated with 500 $\mu \mathrm{M}$ OA cells for 1 day was approximately 2.2-fold of the activity in control cells. A similar result was obtained when the cells were incubated with $30 \mathrm{mM}$ glucose for 2 days (1.9-fold increase compared to the control cells; Fig. 1A).

PARP activation catalyzed acceptor proteins by using NAD, which is an important metabolite in the modulation of cellular metabolism; therefore, we examined the intracellular NAD content of HepG2 cells treated with OA and glucose. Cells treated with $500 \mu \mathrm{M}$ OA for 1 day exhibited a $25 \%$ decrease in cellular NAD level, which was similar to the decrease observed in cells treated with $30 \mathrm{mM}$ glucose for 2 days (24\% decrease; Fig. 1B). 
Fig. 1. Effects of oleic acid (OA) and glucose on poly (ADP)-ribose polymerase (PARP) activity and intracellular nicotinamide adenine dinucleotide (NAD) content. HepG2 cells were cultured with $5.5 \mathrm{mM}$ glucose (control) or 30 $\mathrm{mM}$ glucose for the indicated days, or treated with different concentrations of bovine serum albumin (BSA; control) or OA for 1 day. PARP expression and activity (PAR) were examined by western blotting. Both $\mathrm{OA}$ and $30 \mathrm{mM}$ glucose increased PARP activity (PAR), without markedly changing PARP expression (A). Intracellular NAD content was decreased after treatment with OA or 30 $\mathrm{mM}$ glucose (B). Actin was used as control to confirm equal loading of proteins. Bars represent the mean $\pm \mathrm{SD}(\mathrm{n}=3)$. ${ }^{*} \mathrm{P}<0.05$ versus control.

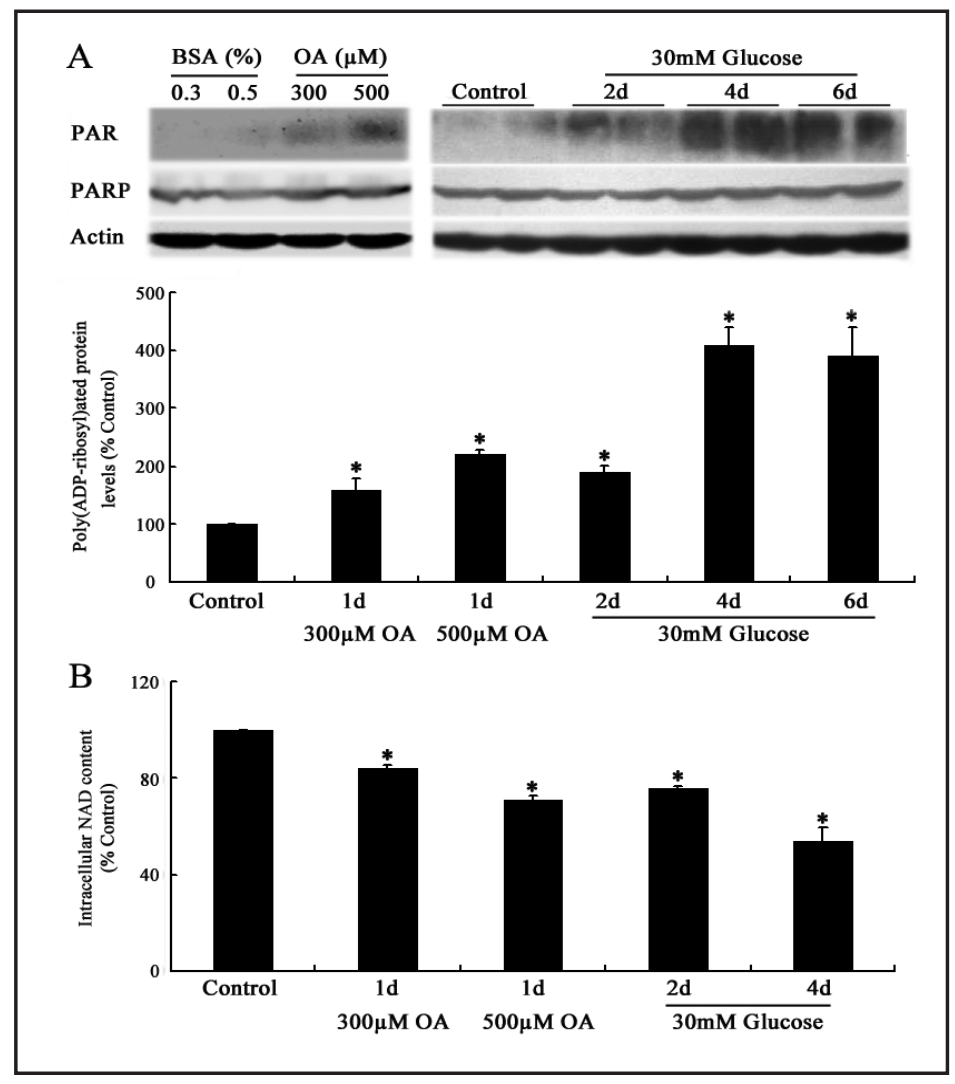

Effects of OA and glucose on SIRT1 expression and insulin sensitivity

SIRT1 expression in cells treated with $300 \mu \mathrm{M}$ or $500 \mu \mathrm{M}$ OA for 1 day was significantly lower (35\% and $42 \%$ decrease, respectively) than the expression in BSA-treated cells (controls; Fig. 2A). The expression of SIRT1 decreased by $11 \%$ and $40 \%$ in cells treated with $30 \mathrm{mM}$ glucose for 2 and 4 days, respectively, compared with the expression levels in cells treated with $5.5 \mathrm{mM}$ glucose (Fig. 2A). In addition to SIRT1 expression, AMPK activity is affected by the increased PARP activity in cells showing glucose toxicity [4]. The actions of SIRT1 and AMPK are always interlinked in cellular metabolism. Therefore, we evaluated AMPK expression and activity in our experimental models. Cells treated with $500 \mu \mathrm{M}$ OA for 1 day exhibited a slight decrease in AMPK activity (20\% decrease compared to the controls; Fig. 2B). In contrast, cells treated with $30 \mathrm{mM}$ glucose for 2 days showed a $22 \%$ decrease in AMPK phosphorylation compared to that in the control group (Fig. 2B).

To determine whether fatty acids or glucose affected the insulin-signaling pathway and contributed to insulin sensitivity, we compared the levels of phosphorylated insulin receptor in insulin-stimulated cells treated with different concentrations of OA or $30 \mathrm{mM}$ glucose. In cells treated with $500 \mu \mathrm{M} \mathrm{OA}$ for 1 day, the phosphorylation of insulin receptor in response to insulin stimulation was reduced by approximately $52 \%$, compared to the control group (Fig. 2C). Treatment with $30 \mathrm{mM}$ glucose for 4 days reduced insulin receptor phosphorylation by approximately 58\%, compared to the level in the control group (Fig. 2C). Thus, in HepG2 cells, the reduction of SIRT1 expression and insulin receptor phosphorylation induced by OA occurred earlier than that induced by glucose.

\section{Effects of the combination of OA and glucose on PARP and SIRT1 expression}

High glucose concentrations and FFAs always coexist in insulin-resistant states; we therefore examined the effect of the combination of $\mathrm{OA}$ and glucose on the expression of PARP and SIRT1. PARP expression did not change after treatment with $30 \mathrm{mM}$ glucose and $500 \mu \mathrm{M}$ OA for 1 day. However, PARP activity was higher in cells treated with this glucose and 


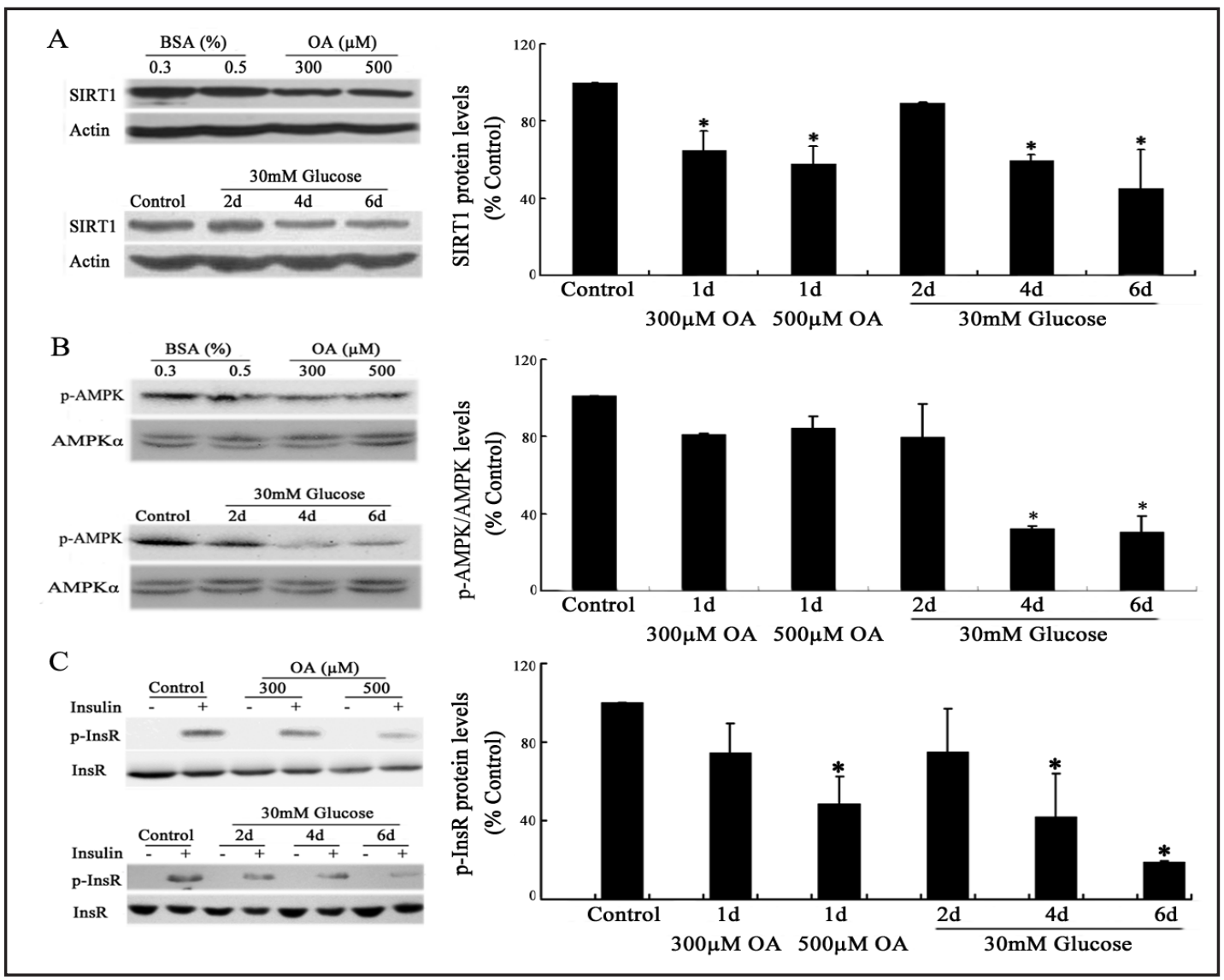

Fig. 2. Effects of oleic acid (OA) and glucose on SIRT1 expression and insulin sensitivity. HepG2 cells were treated as in Figure 1. Both SIRT1 expression (A) and insulin receptor phosphorylation (p-InsR) (C) were decreased in HepG2 cells treated with $500 \mu \mathrm{M}$ OA for 1 day or with $30 \mathrm{mM}$ glucose for 4 days. The phosphorylation of AMPK decreased significantly in HepG2 cells treated with high concentrations of glucose (B). BSA, bovine serum albumin. Actin, AMPK $\alpha$ and insulin receptor (InsR) were used as control to confirm equal loading of proteins. Bars represent the mean $\pm \mathrm{SD}(\mathrm{n}=3)$. ${ }^{*} \mathrm{P}<0.05$ versus control.

OA combination than in cells treated with either OA or glucose alone $(\mathrm{P}<0.05$; Fig. 3). In contrast, SIRT1 expression was significantly lower in cells treated with both OA and glucose than in cells treated with either one of these compounds ( $<<0.05$; Fig. 3).

\section{Effects of T0901317 on the modulation of SIRT1 expression}

To explore the pathways involved in the repression of SIRT1 expression by OA, we examined the expression of the nuclear receptor $\operatorname{LXR} \alpha$, which is a target of fatty acids in cells. The LXR $\alpha$ protein was markedly upregulated in cells stimulated with $300 \mu \mathrm{M}$ or 500 $\mu \mathrm{M}$ OA for 1 day (Fig. 4A). To determine whether OA affected SIRT1 expression through LXR $\alpha$, we used the LXR $\alpha$ activator T0901317 to stimulate HepG2 cells and then examined the expression of SIRT1. Cells treated with $5 \mu \mathrm{M}$ T0901317 for 1 day showed significantly increased expression of LXR $\alpha$ and decreased levels of SIRT1 protein (Fig. 4B and C). However, T0901317 had no effect on SIRT1 mRNA level (Fig. 4D).

\section{Effects of $O A$ and glucose on cell viability}

Both glucotoxicity and lipotoxicity have been reported to affect cell survival; therefore, we analyzed the effect of $\mathrm{OA}$ and glucose on cell viability. Compared to the control cells, 


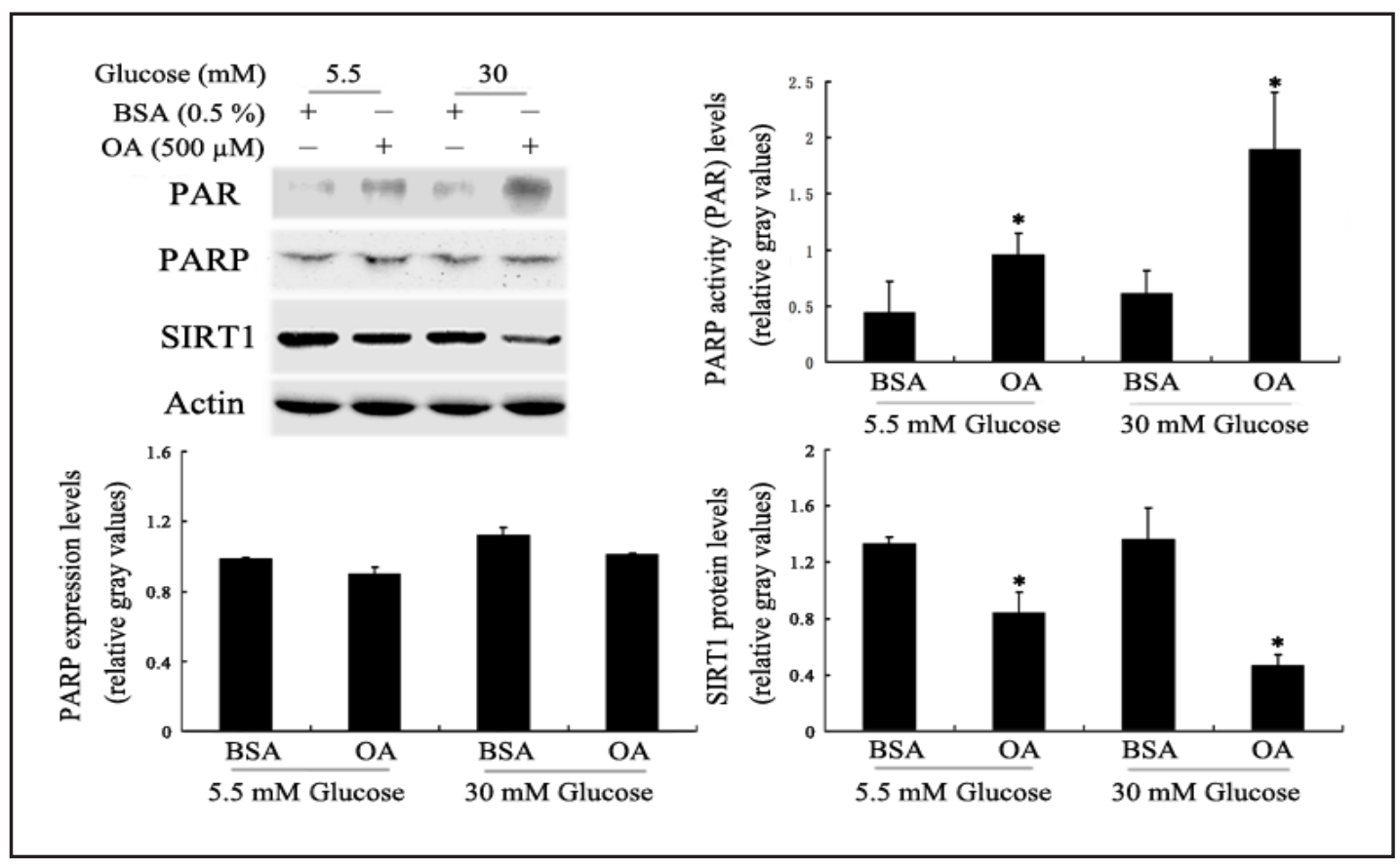

Fig. 3. Effects of the combination of oleic acid (OA) and glucose on poly (ADP)-ribose polymerase (PARP) and SIRT1 expression. HepG2 cells were stimulated using $500 \mu \mathrm{M}$ OA with $5.5 \mathrm{mM}$ glucose or $30 \mathrm{mM}$ glucose for 1 day. PARP expression, PARP activity (PAR) and SIRT1 expression were detected by western blotting. The combination of OA and $30 \mathrm{mM}$ glucose significantly increased PARP activity and decreased SIRT1 expression. BSA, bovine serum albumin. Actin was used as control to confirm equal loading of proteins. Bars represent the mean $\pm \mathrm{SD}(\mathrm{n}=3) .{ }^{*} \mathrm{P}<0.05$ versus $\mathrm{BSA}$ with $5.5 \mathrm{mM}$ glucose.

Fig. 4. Effects of T0901317 on the modulation of SIRT1 expression. Oleic acid (OA) induced LXR $\alpha$ expression in HepG2 cells (A). The LXRa activator T0901317 (5 $\mu \mathrm{M})$ specifically increased LXR $\alpha$ expression (B) and decreased SIRT1 expression (C). The effect of T0901317 on the SIRT1 mRNA level was not significant (D). BSA, bovine serum albumin; DMSO, dimethyl sulfoxide. Bars represent the mean \pm SD $(\mathrm{n}=$ 3). ${ }^{*} \mathrm{P}<0.05$ versus HepG2 cells treated with BSA (A). ${ }^{*} \mathrm{P}<0.05$ versus HepG2 cells treated with DMSO (B-D).

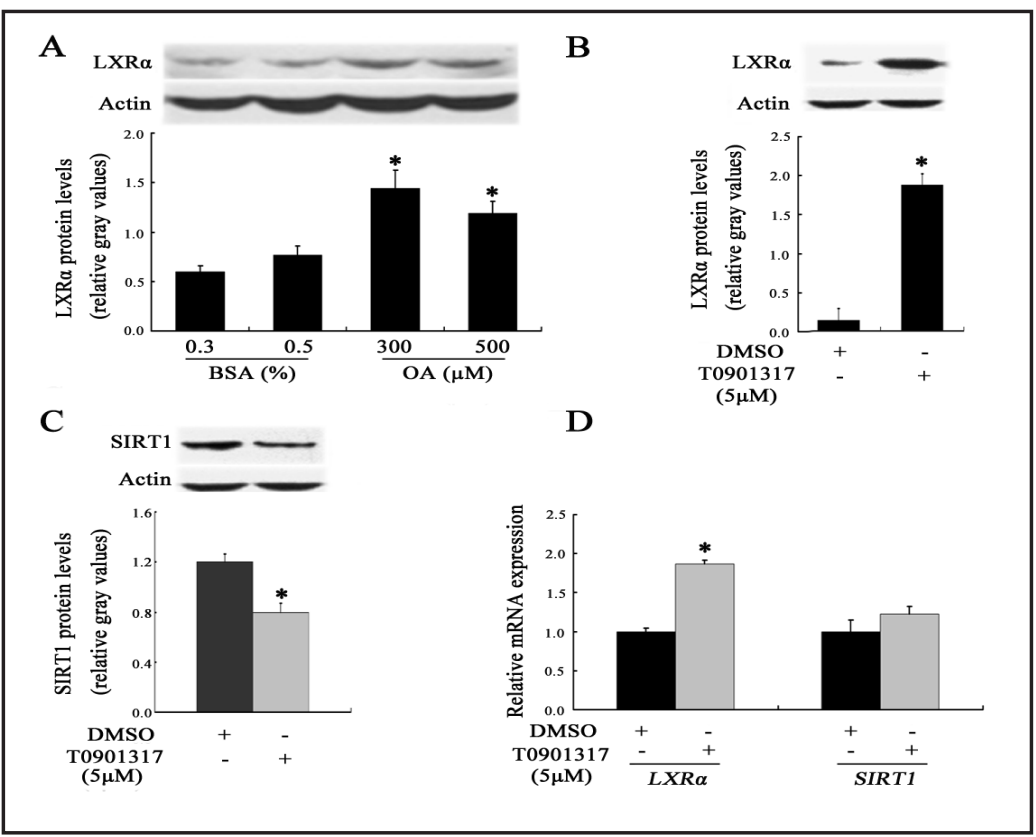

approximately $82 \%$ of cells treated with $500 \mu \mathrm{M} \mathrm{OA}$ survived after 1 day and $77 \%$ of cells treated with $30 \mathrm{mM}$ glucose survived after 4 days (Fig. 5). These results indicated that although $\mathrm{OA}$ and glucose induced cell dysfunction, they had little effect on the viability of HepG2 cells. 
Fig. 5. Effects of oleic acid (OA) and glucose on cell viability. HepG2 cells were cultured with $5.5 \mathrm{mM}$ glucose (control) or $30 \mathrm{mM}$ glucose for the indicated days, or treated with different concentrations of bovine serum albumin (BSA; control) or OA for 1 day. Both $\mathrm{OA}$ and $30 \mathrm{mM}$ glucose slightly affected the viability of HepG2 cells. Bars represent the mean \pm SD $(n=3)$. ${ }^{*} \mathrm{P}<0.05$ versus control.

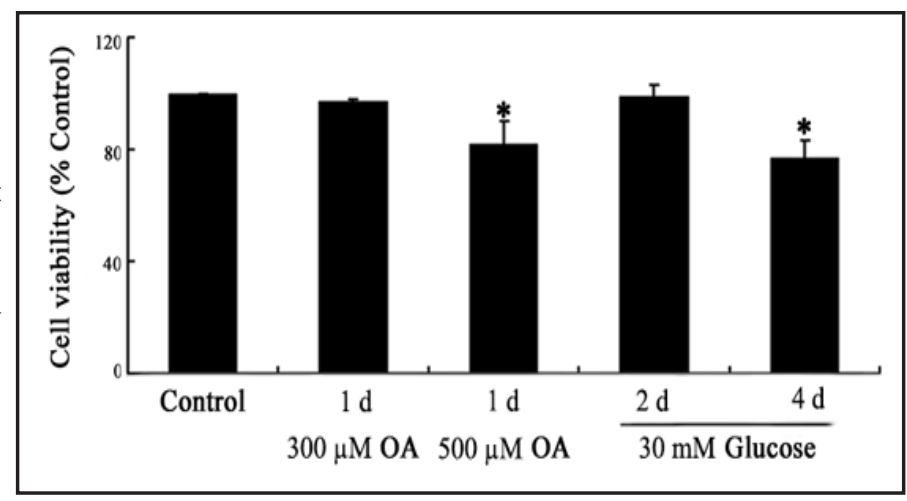

\section{Discussion}

Insulin resistance in type 2 diabetes results from a combination of hyperglycemia and elevated FFA concentration $[11,12]$; however, the precise intracellular mechanisms remain to be elucidated. Our previous study demonstrated that PARP activation decreased insulin sensitivity through NAD depletion and SIRT1 inhibition, which provided a new mechanism of glucotoxicity in the liver [4]. Herein, we proved that the PARP-NAD-SIRT1 pathway is also involved in lipotoxicity in hepatocytes. The results of this study showed that PARP was activated in response to OA stimulation, and this was accompanied by NAD depletion and decreased SIRT1 expression in HepG2 cells.

Thus, both glucotoxicity and lipotoxicity can decrease SIRT1 expression by depleting the intracellular NAD pools due to extensive use of NAD as a substrate for PARP activation in hepatocytes. Therefore, we compared the effects of $\mathrm{OA}$ and glucose on intracellular SIRT1 expression, PARP activity and NAD levels. The results showed that the extent of PARP activation and NAD reduction in cells treated with $500 \mu \mathrm{M}$ OA for 1 day was similar to that in cells cultured with $30 \mathrm{mM}$ glucose for 2 days, while the extent of reduction in SIRT1 expression was similar in cells treated with $500 \mu \mathrm{M}$ OA for 1 day and cells cultured with $30 \mathrm{mM}$ glucose for 4 days. We also compared the effect of glucose and OA on insulin sensitivity. The reduction of insulin receptor phosphorylation in cells treated with $500 \mu \mathrm{M}$ OA for 1 day was equal to that in cells incubated with $30 \mathrm{mM}$ glucose for 4 days. All the data demonstrated that the cellular dysfunction induced by $\mathrm{OA}$ occurred earlier than that induced by glucose. This conclusion was consistent with an in vivo experiment which showed that rapid $(<2 \mathrm{~h})$ lipid infusion decreases myocellular glucose utilization in rats [13], suggesting that lipid stimulation for a very short time could change cellular function. Different environmental stimuli induce different reactions in live cells. Our data demonstrated that for identical treatment times, cells reacted more strongly to treatment with high FFA levels than to treatment with high glucose concentrations. This suggested that lipotoxicity may be more rapid and more potent than glucotoxicity in the liver. Moreover, we found that the combination of $\mathrm{OA}$ and glucose exacerbated the cellular injury.

We next investigated the possible mechanisms underlying SIRT1 modulation by OA in hepatocytes. Since SIRT1 is an NAD-dependent enzyme, changes in intracellular NAD content could affect SIRT1 activity. Treatment with $500 \mu \mathrm{M}$ OA for 1 day produced a $25 \%$ reduction in NAD levels, which was not enough to explain the $42 \%$ decrease observed in SIRT1 expression. Therefore, other mechanisms of SIRT1 modulation may be responsible for the effects induced by OA stimulation. Many studies have focused on the role of nuclear receptors in the modulation of lipid metabolism [14]. LXR $\alpha$, a nuclear receptor, plays an important role in lipid homeostasis and is induced by OA in primary hepatocytes [15]. Similar results were obtained in our study: OA increased LXR $\alpha$ expression in HepG2 cells. We found that the LXR $\alpha$ activator T0901317 decreased SIRT1 protein expression in HepG2 cells. This suggested that FFAs modulate SIRT1 expression in hepatocytes through multiple ways. 
However, T0901317 did not affect the SIRT1 mRNA level, suggesting that the modulation of SIRT1 expression by T0901317 is regulated via a post-transcriptional mechanism. The precise mechanism, however, needs to be investigated further.

There are many types of FFAs in the body. The concentrations of C16:0 (palmitic acid), C18:0 (stearic acid) and C18:1n-9 (OA) are higher in patients with type 2 diabetes than in healthy controls $[6,16]$. However, different types of FFAs play differing roles in the progression of diabetes. $\mathrm{OA}$ is more steatogenic but less apoptotic than palmitic acid in hepatocyte cultures [17]. We primarily discussed the alteration of cellular function in response to different nutritional environments other than those causing cell death. Therefore, we selected OA to test the effects of high concentrations of FFAs on HepG2 cells. We found that both $\mathrm{OA}$ and high glucose concentrations had little effect on the viability of HepG2 cells.

The concentration of OA in our study was lower than that observed in type 2 diabetes mellitus (C18:1, 2-3 mM) [16]; in contrast, the concentration of glucose in our study was much higher than that observed in type 2 diabetes (fasting plasma glucose $>7 \mathrm{mM}$ ). Although the $\mathrm{OA}$ concentration used in this study was lower than the in vivo concentration seen in diabetic patients, it induced a stronger effect than that induced by the glucose concentration, which was higher than the in vivo concentration seen in diabetes. This indirectly proves that lipotoxicity is more potent than glucotoxicity in hepatocytes.

In the present study, we assessed the alterations in PARP activity, SIRT1 expression and insulin receptor phosphorylation induced by $\mathrm{OA}$, and compared these effects with those of high glucose concentrations in hepatocytes. Our results showed that for the same treatment duration, $\mathrm{OA}$ induced a greater degree of cellular dysfunction than high glucose concentrations in HepG2 cells. Since SIRT1 plays key roles in intracellular metabolism, we next studied the modulation of SIRT1 expression in order to elucidate the pathophysiological processes underlying type 2 diabetes. We found that both PARP and LXR $\alpha$ were activated by OA and affected SIRT 1 expression in hepatocytes. The above results show that lipids modulate cellular function through multiple ways, resulting in a more rapid and more potent toxic effect than that induced by glucose in the liver. Relative quantitative comparison between lipotoxicity and glucotoxicity may improve our understanding of the pathophysiological processes of diabetes and provide targets for medical treatment.

\section{Acknowledgements}

We thank EY Zhang and Yan Zhang for the technical assistance. This work was supported by Grants from National Natural Science Foundation of China (No: 81100570 and No: 30971395); Ministry of Science and Technology (2012BAI10B01); Fundamental Research Funds for the Central Universities (105561gk).

\section{References}

1 Robertson RP, Harmon J, Tran PO, Poitout V: Beta-cell glucose toxicity, lipotoxicity, and chronic oxidative stress in type 2 diabetes. Diabetes 2004; 1:S119-124.

- Nakajima K, Yamauchi K, Shigematsu S, Ikeo S, Komatsu M, Aizawa T, Hashizume K: Selective attenuation of metabolic branch of insulin receptor down-signaling by high glucose in a hepatoma cell line, HepG2 cells. J Biol Chem 2000;275:20880-20886.

3 Gao Z, Zhang X, Zuberi A, Hwang D, Quon MJ, Lefevre M, Ye J: Inhibition of insulin sensitivity by free fatty acids requires activation of multiple serine kinases in 3T3-L1 adipocytes. Mol Endocrinol 2004;18:20242034.

4 Pang J, Gong H, Xi C, Fan W, Dai Y, Zhang TM: Poly(ADP-ribose) polymerase 1 is involved in glucose toxicity through SIRT1 modulation in HepG2 hepatocytes. J Cell Biochem 2011;112:299-306. 


\section{Cellular Physiology Cell Physiol Biochem 2013;32:719-727 and Biochemistry

5 de Murcia JM, Niedergang C, Trucco C, Ricoul M, Dutrillaux B, Mark M, Oliver FJ, Masson M, Dierich A, LeMeur M, Walztinger C, Chambon P, de Murcia G: Requirement of poly(ADP-ribose) polymerase in recovery from DNA damage in mice and in cells. Proc Natl Acad Sci USA 1997;94:7303-7307.

6 Yi LZ, He J, Liang YZ, Yuan DL, Chau FT: Plasma fatty acid metabolic profiling and biomarkers of type 2 diabetes mellitus based on GC/MS and PLS-LDA. FEBS Lett 2006;580:6837-6845.

-7 Azevedo-Martins AK, Monteiro AP, Lima CL, Lenzen S, Curi R: Fatty acid-induced toxicity and neutral lipid accumulation in insulin-producing RINm5F cells. Toxicol In Vitro 2006;20:1106-1113.

-8 Rodgers JT, Lerin C, Haas W, Gygi SP, Spiegelman BM, Puigserver P: Nutrient control of glucose homeostasis through a complex of PGC-1alpha and SIRT1. Nature 2005;434:113-118.

-9 Picard F, Kurtev M, Chung N, Topark-Ngarm A, Senawong T, Machado De Oliveira R, Leid M, McBurney MW, Guarente L: Sirt1 promotes fat mobilization in white adipocytes by repressing PPAR-gamma. Nature 2004;429:771-776.

10 Sun C, Zhang F, Ge X, Yan T, Chen X, Shi X, Zhai Q: SIRT1 improves insulin sensitivity under insulin-resistant conditions by repressing PTP1B. Cell Metabolism 2007;6:307-319.

11 Groop LC, Bonadonna RC, Del Prato S, Ratheiser K, Zyck K, Ferrannini E, DeFronzo RA: Glucose and free fatty acid metabolism in non-insulin-dependent diabetes mellitus. Evidence for multiple sites of insulin resistance. J Clin Invest 1989;84:205-213.

12 Groop L, Saloranta C, Shank M, Bonadonna RC, Ferrannini E, DeFronzo RA: The role of free fatty acid metabolism in the pathogenesis of insulin resistance in obesity and noninsulin-dependent diabetes mellitus. J Clin Endocrinol Metab 1991;72:96-107.

13 Jucker BM, Rennings AJM, Cline GW, Shulman GI: 13C and 31P NMR studies on the effects of increased plasma free fatty acids on intramuscular glucose metabolism in the awake rat. J Biol Chem 1997;272:10464-10473.

-14 Peet DJ, Turley SD, Ma W, Janowski BA, Lobaccaro JM, Hammer RE, Mangelsdorf DJ: Cholesterol and bile acid metabolism are impaired in mice lacking the nuclear oxysterol receptor LXR a. Cell 1998;93:693-704.

-15 Tobin KA, Steineger HH, Alberti S, Spydevold O, Auwerx J, Gustafsson JA, Nebb HI: Cross-talk between fatty acid and cholesterol metabolism mediated by liver X receptor-alpha. Mol Endocrinol 2000;14:741-752.

-16 Liu L, Li Y, Guan C, Li K, Wang C, Feng R, Sun C: Free fatty acid metabolic profile and biomarkers of isolated post-challenge diabetes and type 2 diabetes mellitus based on GC-MS and multivariate statistical analysis. J Chromatography B 2010;878:2817-2825.

17 Ricchi M, Odoardi MR, Carulli L, Anzivino C, Ballestri S, Pinetti A, Fantoni LI, Marra F, Bertolotti M, Banni S, Lonardo A, Carulli N, Loria P: Differential effect of oleic and palmitic acid on lipid accumulation and apoptosis in cultured hepatocytes. J Gastroenterol Hepatol 2009;24:830-840. 